\title{
An Integrated Token-Based Algorithm for Scalable Coordination
}

\author{
Yang $\mathrm{Xu}^{+}$, Paul Scerri*, Bin Yu*, Steven Okamoto*, Michael Lewis ${ }^{+}$and Katia Sycara* \\ + University of Pittsburgh, * Carnegie Mellon University \\ yxu@sis.pitt.edu, \{pscerri, byu, sokamoto\}@cs.cmu.edu, ml@sis.pitt.edu, katia@cs.cmu.edu
}

\begin{abstract}
Efficient coordination among large numbers of heterogeneous agents promises to revolutionize the way in which some complex tasks, such as responding to urban disasters can be performed. However, state of the art coordination algorithms are not capable of achieving efficient and effective coordination when a team is very large. Building on recent successful token-based algorithms for task allocation and information sharing, we have developed an integrated and efficient approach to effective coordination of large scale teams. We use tokens to encapsulate anything that needs to be shared by the team, including information, tasks and resources. The tokens are efficiently routed through the team via the use of local decision theoretic models. Each token is used to improve the routing of other tokens leading to a dramatic performance improvement when the algorithms work together. We present results from an implementation of this approach which demonstrates its ability to coordinate large teams.
\end{abstract}

\section{INTRODUCTION}

Efficient and flexible coordination among large numbers of robots, agents and people promises to revolutionize the achievement of complex and distributed tasks. In domains such as disaster response [9], the military [20] and business organizations [6], decentralized cooperative coordination can dramatically reduce costs and improve efficiency while lowering risks and improving safety. In these applications, a large number of heterogeneous agents need to coordinate in a dynamic, uncertain environment and adjust their activities according to the status of the team and their teammates. Typically, coordination requires tasks including plan monitoring, information delivery, role allocation and resource sharing. While rapid progress has been made in developing coordination algorithms [7], teamwork algorithms that scale to large numbers of agents while remaining efficient, distributed and flexible are not yet available.

Previous work on coordination has typically focused on only one specific coordination task, e.g. role allocation [15]

Permission to make digital or hard copies of all or part of this work for personal or classroom use is granted without fee provided that copies are not made or distributed for profit or commercial advantage and that copies bear this notice and the full citation on the first page. To copy otherwise, to republish, to post on servers or to redistribute to lists, requires prior specific permission and/or a fee.

AAMAS'05, July 25-29, 2005, Utrecht, Netherlands.

Copyright 2005 ACM 1-59593-094-9/05/0007 ...\$5.00. or planning [8], precluding the use of knowledge from other aspects of coordination being used to improve the performance of that algorithm. For example, results of the task allocation process have not been used to guide resource allocation, although intuitively they will improve the search. Moreover, even algorithms designed for single coordination tasks do not scale well to very large teams [14, 24] because of infeasible requirements such as requiring accurate models of all team members [14]. Algorithms that are scalable, often rely on swarm-like behavior that, while robust, can be very inefficient [3]. Other approaches, e.g., using an auctioneer [8], require some degree of centralization which is not always desirable.

In this paper, we present an integrated and scalable approach to coordinating a large number of heterogeneous agents. Three novel ideas underlie this approach. The first idea is to encapsulate all coordination interactions, including information, assignable tasks and sharable resources within tokens. The agent holding the token has exclusive control over whatever is represented by that token, hence tokens provide a type of access control. Agents either keep tokens or pass them to teammates. For example, an agent holding a resource token has exclusive access to the resource represented by that token and passes the token on to transfer access to that resource. The resulting movement of tokens implements the coordination by distributing information, resources and tasks with low communication overhead.

The second novel idea is for agents to use local decision theoretic models to determine when and where to pass tokens. When an agent passes a token to another agent, that exchange is used to refine local models of the team. These models are used in a decision theoretic way to determine whether and where to forward any token the agent currently holds, so as to maximize the expected utility of the team. Informally, agents will try to pass tokens to where they help team performance the most by inferring from their local models which team member will either have use for the information, resource or task represented by the token or be in the best position to know who will. A logical static network across the team, limits agents to forwarding tokens to their neighbors in this network. As a result an agent directly receives tokens from only a small number of neighbors in the network and can thus build better models of those agents. By ensuring that the network has a small world property $[22]$, i.e., the distance between any two nodes in the network is small, the effect of these better models outweighs the additional number of "hops" a token might need to take to get where it is required. 
The third novel idea in this work is to leverage all available information for creating models of the team, specifically using the movement of one token to inform the movement of other tokens. This synergistically integrates the execution of key coordination algorithms in a way not done before. For example, tokens representing resources useful for a particular task should be passed to the same agent as the token representing that task was. Intuitively, making use of the relationship between tokens, each coordination task becomes more efficient because it focuses its search based on the progress of other coordination tasks.

In the remainder of this paper we describe how tokens are routed around the network to maximize the expected utility of the team. Specifically, we begin with a Markov Decision Process (MDP) model based on the fully observation of team state then make a series of approximations to develop efficient, local reasoning for routing the tokens. Our experiments show that the local routing models lead to a dramatic improvement in coordination performance. Moreover, excluding any type of token from the development of the local reasoning models decreases performance. Notice that this paper focuses on the local routing models and builds on previously described individual token-based algorithms [15, 18, 21, 23].

\section{LARGE SCALE COORDINATION}

In this section, we provide a detailed model of the organization and coordination problem for the team.

\subsection{Problem Description}

Coordination is required between a team $A=\left\{\alpha_{1}, \alpha_{2}, \ldots\right.$, $\left.\alpha_{n}\right\}$ of agents that share a top level common goal $G$ (as in [19]). Achieving $G$ requires achieving a number of sub-goals $\left\{g_{1}, g_{2}, \ldots, g_{i}, \ldots\right\}$. When sub-goal $g_{i}$ is satisfied the team receives a reward reward $_{i}$. For example, sub-goals of a high level goal to respond to a disaster might be to extinguish fires and provide medical attention to injured civilians. To satisfy sub-goals, the team follows plan templates Plan = $\left\{\right.$ plan $_{1}$, plan $_{2}, \ldots$, plan $\left._{i}, \ldots\right\}$ represented in a library. Each template $i$ includes four parts and is written as plan $i=<$ $g_{i}$, conditions $_{i}$, roles $_{i}$, reward $_{i}>$. The first element is the sub-goal $g_{i}$; the second is the conditions under which it is applicable, condition $_{i}=$ event $_{1} \cap$ event $_{2} \cap$... nevent $t_{l}$; the third element is the individual roles roles $_{i}=\left\{r_{1}, r_{2}, \ldots, r_{k}\right\}$ which are required to achieve $g_{i}$ and the last part, the reward $d_{i}$ is to be received by the team on successful satisfaction of $g_{i}$. Each role $r_{i}=<$ task $_{i}$, ability $_{i}$, resource $_{i}>$ is represented by its task, i.e., a description of the actual thing to be done, the capabilities required to perform that task and the resources needed to perform the role.

For example, a fire fighting template can be defined as: $<$ plan $_{\text {fire }}=($ Fight fire at location $\mathrm{X})$, (Fire alarm at $\mathrm{X} \cap$ Smoke at $\mathrm{X}),\left\{r_{1}, r_{2}, r_{3}\right\},(100)>$. This template requires two conditions before it is initiated: a fire alarm and smoke. After this plan is initiated, three roles, $\left\{r_{1}, r_{2}, r_{3}\right\}$ need to be assigned and a reward 100 will be credited to the team. The three roles in this template are: driving the fire truck, fighting the fire and searching for victims, i.e., $r_{1}=<$ (Driving the fire truck), (Skillful in driving truck), (Fire truck) $>, r_{2}=<$ (fighting the fire), (Have training in fire fighting), (Hose, water) $>$ and $r_{3}=<$ (Searching for victims), (None), (Breathing equipment) $>$. To perform $r_{1}$, an agent is required to be able to drive and have access to a fire truck

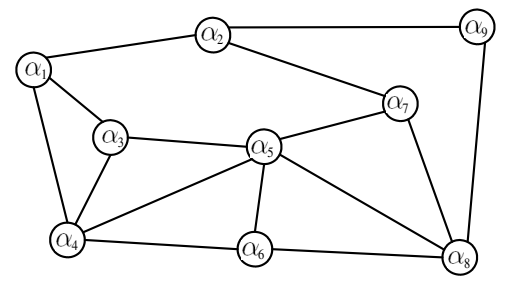

Figure 1: An example of a subset of a typical acquaintance network.

which is an exclusive resource.

\subsection{Acquaintance Network}

We arrange the team as an acquaintance network. The acquaintance network is a graph $G=(A, N)$, where A is the team of agents and $\mathrm{N}$ is the set of links between agents. Specifically, for $\alpha_{i}, \alpha_{j} \in A,<\alpha_{i}, \alpha_{j}>\in N$ denotes that $\alpha_{i}$ and $\alpha_{j}$ are acquaintances and are able to exchange tokens directly. $n(\alpha)$ is defined as all the acquaintances of agent $\alpha$. Note that $n(\alpha)<<|A|$. We additionally require that the acquaintance network be a small world network, which means a relatively small number of links separate any two agents than regular grid network. Previous work has shown that such networks lead to better performance of token-based algorithms [23]. A subset of a typical acquaintance network for a large team is shown as Figure 1. In the Figure, each node represents a team member and when pairs of agents are connected by a line, they can exchange tokens with each other directly.

\section{TOKENS FOR COORDINATION}

Token-based algorithms for specific tasks have been developed by us and others and have been shown to be effective for specific coordination tasks [23, 15]. Control information is included in the token to help the agents determine what to do within the token. For example, for information sharing the control information is the number of "hops" a token can move before stopping [23]. For task allocation, the control information is the minimum capability an agent must have to accept a task [15]. However, while these algorithms share the important common feature of being based on tokens, they operate separately. In this paper, we generalize and integrate token-based approaches to make a complete approach to coordination.

Let $\Gamma=\left\{\Delta_{1}, \Delta_{2}, \ldots, \Delta_{m}\right\}$ be all types of coordination tokens. These tokens can be classified into three basic types: information tokens, roles tokens and resources tokens. Each token $\Delta_{i}$ is defined as a tuple with four elements: $\Delta_{i}=<$ Type, Coordination, Path, Threshold $>$. Type $=\{$ inf, role, res $\}$ denotes the type of coordination it contains information, role or resource respectively. Coordination captures the specific coordination element represented by this token. In the case of an information token, it is the information to be shared. In the case of a resource token, it is a description of the resource to which this token grants exclusive access. In the case of a role token, it is a description of the task for which the accepter of this token is responsible. Path records the route the token has taken through the network. $\Delta$.path is also used as stop condition for information and role tokens when $\mid \Delta$.path $\mid>T T L$ where $T T L$ is empirically set to 
be the maximum number of "hops" that $\Delta$ is allowed to be passed. Threshold generalizes the control information for resource and role tokens but is not required for information tokens. An agent may keep a resource if its need for that resource is greater than the token's threshold. Determining an agent's requirement for a resource is outside of the scope of this paper. While an agent holds a resource token $\Delta$, $\Delta$.threshold slowly increases up to some maximum. When the token is passed, $\Delta$.threshold is decreased to avoid the token being passed indefinitely. This mechanism ensures that resources can flow through the team resource. For example, to coordinate a team of Unmanned Aerial Vehicles (UAVs) holding tokens representing airspace, might be forced to relinquish tokens to the longest held regions as their thresholds increased unless the airspace was critical. Similarly, a role token $\Delta$ will be accepted by an agent whose capability is greater than $\Delta$.threshold and its threshold will be decreased if not been accepted. The role allocation algorithm is described in detailed in [15].

\section{ROUTING TOKENS}

Token-based coordination is a process by which agents attempt to maximize the overall team reward by moving tokens around the team. If an agent were to know the exact state of the team, it could use an MDP to determine the expected utility maximizing way to move tokens. Unfortunately, it is infeasible for an agent to know the complete state, however, [14] it is illustrative to look at how tokens would be passed if it were feasible. Then, by dividing the monolithic joint activity into a set of actions that can be taken by individual agents, we can decentralize the token routing process where distributed agents, in parallel, make independent decisions of where to pass the tokens they currently hold. Thus, we effectively break a large coordination problem into many small ones.

\subsection{MDP Model for Complete Team State}

The basic decision model of agent $\alpha$ for a token $\Delta$ can be written as an MDP $<S$, Action $_{\alpha}, T, R>$. $S$ is the state space and its specific value in time $t$ defined as $s(t)$, Action $_{\alpha}$ is the action space of $\alpha, T: S \times A \rightarrow S$, is the transition function that describes the resulting state $s(t+1) \in S$ when executing $\chi \in$ Action $_{\alpha}$ in $s(t) . R: S \rightarrow \mathbb{R}$ defines the instantaneous reward for being in a specific state. This model can be applied to any agent and any token.

In this case, the state $s(t)$ at time $t$ is modelled as the locations of all tokens across the team and is written as: $s(t)=<<$ Tokens $(\alpha, t), H_{\alpha}(t)>,<$ Tokens $\left(\alpha_{1}, t\right), H_{\alpha_{1}}(t)>$ ,$<$ Tokens $\left(\alpha_{2}, t\right), H_{\alpha_{2}}(t)>, \ldots>$. Tokens $(\alpha, t)$ are all the tokens currently held by $\alpha$ and $H_{\alpha}(t)$ records all the incoming and out-going tokens of $\alpha$ before $t$. For notation convenience, we write Tokens $(\alpha, t)$ as Tokens $(\alpha)$ and $H_{\alpha}(t)$ as $H_{\alpha}$ when there is no ambiguity. Figure 2 shows an example of specific team state $s(t)=<<\operatorname{Tokens}(\alpha), H_{\alpha}>,<$ Tokens $\left(\alpha_{1}\right), H_{\alpha_{1}}>,<$ Tokens $\left(\alpha_{2}\right), H_{\alpha_{2}}>>$ where Tokens $(\alpha)=\left\{\Delta, \Delta_{2}\right\}, H_{\alpha}=\left\{\Delta_{3}\right\}$, Tokens $\left(\alpha_{1}\right)=\left\{\Delta_{5}\right\}$, $H_{\alpha_{1}}=\{\Delta\}$, and Tokens $\left(\alpha_{2}\right)=\left\{\Delta_{4}\right\}, H_{\alpha_{2}}=\emptyset$. Since the tokens represent resources, roles and information, $s(t)$ unambiguously defines who is doing what, with what resources and what information.

Action $_{\alpha}: S \rightarrow(n(\alpha) \cup \alpha)$ is to move $\Delta$ to one of $n(\alpha)$ or keep it for itself. For notation convenience, $\chi \in$ Action $_{\alpha}$ can be written as move $(\Delta, b)$ where $b \in(n(\alpha) \cup \alpha)$. Note, keep

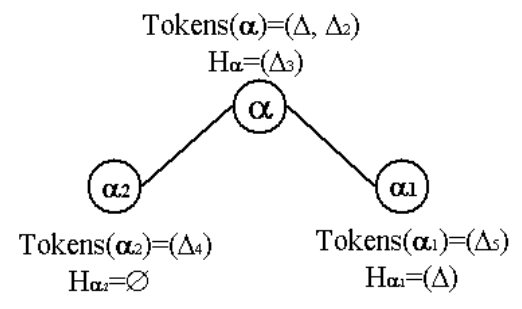

Figure 2: An example showing part of a team. Agent $\alpha$ holds $\Delta, \Delta_{2}$ and has previously had $\Delta_{3}$; $\alpha_{2}$ holds $\Delta_{4}$ while $\alpha_{1}$ holds $\Delta_{5}$ and has previously had $\Delta$.

a token for itself applies when the agent accepts the role or requires the resource or information has propagated sufficiently for across the team. In general, we define a function Acceptable $(\alpha, \Delta)$ to determine whether $\Delta$ should be kept by agent $\alpha$.

$R(s(t))>0$ when at $s(t)$, a sub-goals $g_{i}$ are achieved. Team will be credited an instant rewards value of $R(s(t))=$ reward $_{i}$.

The utility of state $S$ under a policy $\pi$ is defined as

$$
v^{\pi}(s)=\sum_{t=0: \infty}\left(d^{t} \times R(s(t))-t \times \text { commcost }\right)
$$

where commcost is the communication cost and $d<1$ is a predefined discount factor. $v^{*}(s)$ allows the agent to select actions according to optimal policy

$$
\pi^{*}(s(t))=\operatorname{argmax}_{\chi \in \text { Action }_{\alpha}} v^{*}(s(t+1))
$$

By value iteration, $v^{*}(s(t))=\operatorname{argmax}_{\chi \in \text { Action }_{\alpha}}[R(s(t))-$ commcost $\left.+d \times v^{*}(s(t+1))\right]$. This policy tells the agent where to move resources information and roles to maximize the team's expected utility.

We define a matrix $V$ where each element $V[s(t), b]=$ $R(s(t))-$ commcost $+d \times v^{*}(s(t+1))$ when $\chi=\operatorname{move}(\Delta, b)$. Then $V[b]$ represents the expected utilities vector for $\alpha$ to send token $\Delta$ to $b$ at each different state $s(t)$.

\subsection{Local POMDP Model}

Knowing the complete team state is only feasible for small teams. In large teams, agents must make token coordination decisions based on a more limited view of the team. Thus the reasoning must be modelled as a Partially Observable Markov Decision Process (POMDP). Standard POMDP techniques such as [2] and [11] could be used to solve the POMDP to determine optimal token routing. However, for fast routing of tokens, while this local POMDP does tell the agent the optimal action, the computational complexity is still too high for practical applications. However, the POMDP model does provide important hints for how to do a heuristic approach.

The POMDP model is defined as $<S$, Action $_{\alpha}, T, \Theta_{\alpha}, O, R>$. In this case, the observations of agent $\alpha$ are defined as $\Theta_{\alpha}=<$ Tokens $(\alpha, t), H_{\alpha}(t)>$ to include not only the tokens the agent currently holds but also all the previously incoming and out-going tokens (in $H_{\alpha}(t)$ ). The observation function is defined as $O: \Theta_{\alpha} \times S \rightarrow \Omega_{\alpha}$. Belief state $\Omega_{\alpha}$ is a discrete probability distribution vector over the team state $s(t)$ inferred from current local state $\Theta_{\alpha}$. For example, if 
$S=\left\{s_{1}, s_{2}, s_{3}\right\}$ and $\Omega_{\alpha}=[0.6,0.2,0.2], \alpha$ estimates that the probability of $s(t)$ being $s_{1}$ is 0.6 and being $s_{2}$ and $s_{3}$ are 0.2 .

One way of solving a POMDP is via a Q-MDP [11]. Agent $\alpha$ makes use of $V$, see above, to calculate the expected reward vector $E U\left(\Omega_{\alpha}\right)=\Omega_{\alpha} \times V$. For example, if $\alpha$ has acquaintances $b, c$, and $d$ and $E U\left(\Omega_{\alpha}\right)=[5,10,6,4]$, then $E U\left(\Omega_{\alpha}, b\right)=10$ represents the expected utility to send $\Delta$ to $b$ according to the Q-MDP. The locally-optimal policy $\pi^{* *}\left(\Omega_{\alpha}\right)$ is $\operatorname{argmax}_{\chi \in \text { Action }_{\alpha}} E U\left(\Omega_{\alpha}, c\right)$. This is the action the agent should take to maximize expected utility, given that it has an incomplete view of the team state. As in the previous example, passing $\Delta$ to $b$ is the best choice because $E U\left(\Omega_{\alpha}, b\right)=10$ is the maximum value of $E U\left(\Omega_{\alpha}\right)$.

\section{LOCAL HEURISTIC APPROACH}

In this section, we provide a heuristic approach for tokenbased team coordination inspired by the local POMDP. The resulting approach allows fast, efficient routing decisions, without requiring accurate knowledge of the complete state. In the next section, we show that this approach is effective for improving token routing.

\subsection{Local Model}

$P_{\alpha}$ is the decision matrix agent $\alpha$ uses to decide where to move tokens. Each row $P_{\alpha}[\Delta]$ in $P_{\alpha}$ represents a vector that determines the decision where to pass a token $\Delta$ to one of its acquaintances. Specifically, each value $P_{\alpha}[\Delta, b] \rightarrow[0,1], b \in$ $n(\alpha)$ represents $\alpha$ 's decision that the probability of passing token $\Delta$ to an acquaintance $b$ would be the action that maximize team reward. Then our policy $\pi^{* * *}$ for this local model is to choose action $\chi$ to $\operatorname{argmax}_{\chi \in \text { Action }_{\alpha}} P_{\alpha}[\Delta, c]$ where $\chi=\operatorname{move}(\Delta, c)$. Figure 3 shows an example where $P_{\alpha}[\Delta]=[0.6,0.1,0.3]$ and agent $\alpha$ has three acquaintances $\alpha_{1}, \alpha_{2}, \alpha_{3} . P_{\alpha}\left[\Delta, \alpha_{1}\right]=0.6, P_{\alpha}\left[\Delta, \alpha_{2}\right]=0.1, P_{\alpha}\left[\Delta, \alpha_{3}\right]=$ 0.3 and $\pi^{* * *}$ will choose the action move $\left(\Delta, \alpha_{1}\right)$ to pass $\Delta$ to $\alpha_{1}$. The key to this distributed reasoning lies in how the probability model $P_{\alpha}$ for each agent $\alpha$ is updated. If the action indicated by $P_{\alpha}$ matches the optimal policy $\pi^{*}$ from the MDP model, then the team will act optimally.

Initially, agents do not know where to send tokens, but as tokens are received, a model can be developed and better routing decisions made. That is, the model, $P_{\alpha}$ is based on the accumulated information provided by the receipt of previous tokens. For example, when an agent sends a role to an acquaintance that has previously rejected a similar role, the team is potentially hurt because this acquaintance is likely to reject this role too and thus communication bandwidth has been unnecessarily wasted.

From this view point, $P_{\alpha}$ can only depend on $\alpha$ 's history of received tokens, $H_{\alpha}$. The update function $\operatorname{Update}\left(P_{\alpha}[\Delta], \Delta_{i}\right)$ for $P_{\alpha}[\Delta]$ defines the calculation of the probability vector of where to send $\Delta$ based on previously received token $\Delta_{i}$ in $H_{\alpha}$. It will be explained in detail in next section.

Algorithm 1 shows the reasoning of agent $\alpha$ when it receives incoming tokens from its acquaintances via function getToken(sender) (line 2). For each incoming token $\Delta$, function Acceptable $(\alpha, \Delta)$ determines whether the token will be kept by $\alpha$ (line 4$)$. When a resource is kept, its threshold is raised (line 6). If $\alpha$ decides to pass $\Delta$, it will add itself to the path of $\Delta$ (Line 9$)$ and $\operatorname{Update}\left(P_{\alpha}[\Delta], \Delta_{i}\right)$ will update how to send $\Delta$ according to each previously received token $\Delta_{i}$ in $\alpha$ 's history (line 11). If $\Delta$ is a resource or role token,

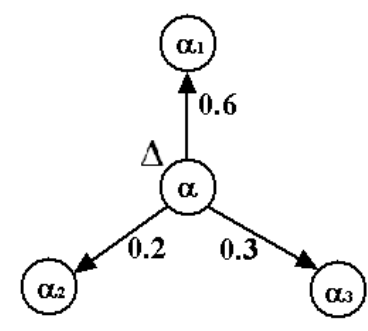

Figure 3: Agent $\alpha$ 's local model for where to send $\Delta$. The probability that $\alpha_{1}$ is the best to send $\Delta$ to is 0.6 and $\alpha$ will pass $\Delta$ to $\alpha_{1}$ according to $\pi^{* * *}$

its threshold will be decreased (line 14). Then $\alpha$ will choose to the best acquaintance to pass the token to according to $P_{\alpha}[\Delta]$ (line 16) and record $\Delta$ in its history, $H_{\alpha}$ (line 18).

Algorithm 1: Decision process for agent $\alpha$ to pass incoming tokens

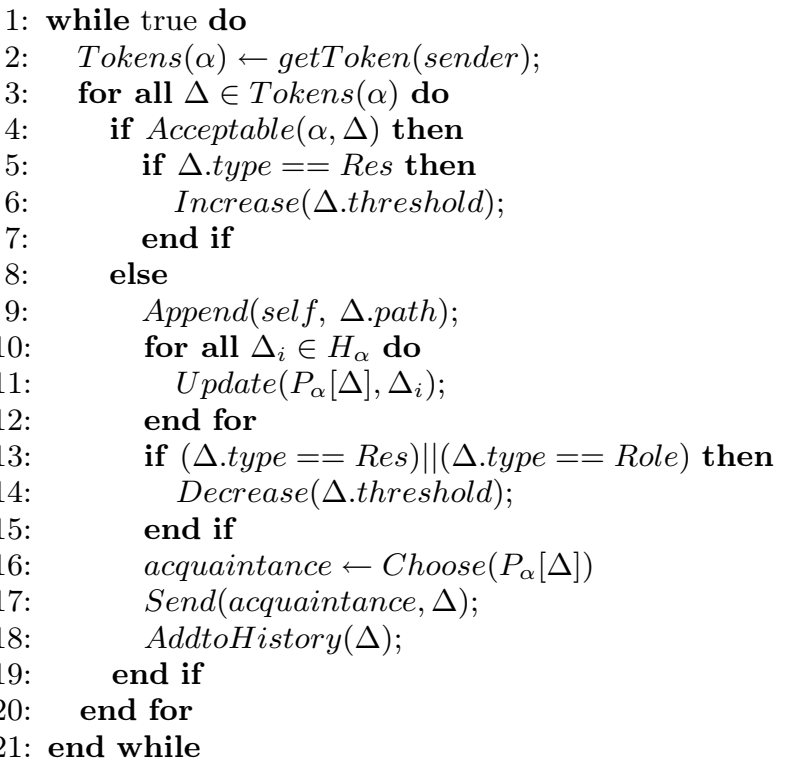

\subsection{Model Update Function}

The effectiveness of the token-based approach depends on how well agents maintain their local models so that tokens are routed to where they lead to the highest gain in expected reward. In this section, we describe an algorithm to update the localized decision model by utilizing previously received tokens. The key is to make use of relationships between tokens, which we refer to as relevance.

Deciding where to send one token based on the receipt of another relies on knowing something about the relationship between the tokens. We quantify this relationship as the Relevance and define the relationship between tokens $\Delta_{i}$ and $\Delta_{j}$ as $\operatorname{Rel}\left(\Delta_{i}, \Delta_{j}\right) . \operatorname{Rel}\left(\Delta_{i}, \Delta_{j}\right)>1$ indicates that an agent with use for $\Delta_{i}$ will often also have use for $\Delta_{j}$, while $\operatorname{Rel}\left(\Delta_{i}, \Delta_{j}\right)<1$ indicates that an agent $\Delta_{i}$ has use for it is unlikely to have use for $\Delta_{j}$. If $\operatorname{Rel}\left(\Delta_{i}, \Delta_{j}\right)=1$ then nothing can be inferred. Details about how relevance is computed to ensure appropriate behavior will be explained in the next section. The update function of $P_{\alpha}\left[\Delta_{j}\right]$ according to $H_{\alpha}$, 
written as $\operatorname{Update}\left(P_{\alpha}\left[\Delta_{j}\right], \Delta_{i}\right)$ where $\Delta_{i} \in H_{\alpha}$ is found by using Bayes' Rule as follows:

$\forall b \in n(\alpha), \forall \Delta_{i} \in H_{\alpha}, d=\operatorname{first}\left(n(a), \Delta_{i}\right.$. path $)$

$\operatorname{Update}\left(P_{\alpha}\left[\Delta_{j}, b\right], \Delta_{i}\right)=$

$\begin{cases}P_{\alpha}\left[\Delta_{j}, b\right] \times \operatorname{Rel}\left(\Delta_{i}, \Delta_{j}\right) & \text { if } \Delta_{i} \neq \Delta_{j}, b=d \\ P_{\alpha}\left[\Delta_{j}, b\right] & \text { if } \Delta_{i} \neq \Delta_{j}, b \neq d \\ P_{\alpha}\left[\Delta_{j}, b\right] \times \varepsilon & \text { if } \Delta_{i}=\Delta_{j}, b \in \Delta_{j} . \text { path } \cap(\alpha)\end{cases}$

where $\operatorname{Update}\left(P_{\alpha}\left[\Delta_{j}, b\right], \Delta_{i}\right)$ is to update the $P_{\alpha}\left[\Delta_{j}, b\right]$ in $P_{\alpha}\left[\Delta_{j}\right]$ according to $\Delta_{i}$ and $\operatorname{first}\left(n(a), \Delta_{i}\right.$.path) extracts from the recorded path of the token the acquaintance of agent $\alpha$ that had the token $\Delta_{i}$ earliest. The first case in this function is the most important. The probability that the sender of previous token $\Delta_{i}$ is the best agent to receive the token $\Delta_{j}$ is updated according to $\operatorname{Rel}\left(\Delta_{i}, \Delta_{j}\right)$. The second case in the equation changes the probability of sending that token to agents other than the sender in a way that ensures the subsequent normalization has the desired effect. Finally, the third case encodes the idea that $\alpha$ should typically not pass a token back from where it came. $P_{\alpha}\left[\Delta_{j}\right]$ is subsequently normalized to ensure that $\sum_{b \in n(\alpha)} P_{\alpha}\left[\Delta_{j}, b\right]=1$.

To see how the updating function works, consider the following example. Supposed agent $\alpha$ has five acquaintances $\{a, b, c, d, e\}$ and $P_{\alpha}\left[\Delta_{j}\right]=[0.1,0.4,0.2,0.2,0.1]$. Moreover, $H_{\alpha}=\left\{\Delta_{i}, \Delta_{k}\right\}, \operatorname{rel}\left(\Delta_{i}, \Delta_{j}\right)=1.2$ and $\operatorname{rel}\left(\Delta_{k}, \Delta_{j}\right)=0.4$. $\Delta_{i}$.path $=\{b, ..\} ; \Delta_{k}$. path $=\{c, ..\} ; \Delta_{j}$. path $=\{e, .$.$\} . If$ currently $\alpha$ holds $\Delta_{j}$, by applying our updating function to $P_{\alpha}\left[\Delta_{j}\right]$, we get the result as $P_{\alpha}\left[\Delta_{j}\right]=[0.12,0.56,0.09,0.23, \varepsilon]$ and $\Delta_{j}$ will be passed to acquaintance $b$.

\subsection{Token Similarity}

When an agent receives two tokens that are relevant to one another they are more likely to be usable in concert to obtain a reward for the team. While infeasibly complex, the POMDP model can suggest how relevance should be defined. If the local policy $\pi^{* * *}$ always matches with $\pi^{* *}, P_{\alpha}[\Delta]$ will be the normalization of $E U\left(\Omega_{\alpha}\right)$.

$$
\forall b \in n(\alpha), \quad P_{\alpha}[\Delta, b]=\frac{E U\left(\Omega_{\alpha}, b\right)}{\sum_{c \in N(\alpha)} E U\left(\Omega_{\alpha}, c\right)}
$$

That is, the largest expected utility for sending a token to an acquaintance should result the highest probability. Following the previous example, if $E U\left(\Omega_{\alpha}\right)=[5,10,6,4]$, then for optimal behavior $P_{\alpha}[\Delta]=[0.2,0.4,0.24,0.16]$.

Now we are in a position to see how the receipt of a token affects the locally optimal policy for routing token $\Delta$ and hence determine how to compute relevance. Suppose that the state estimation of agent $\alpha$ just before a token $\Delta_{\text {pre }}$ arrives is $\Omega_{\alpha}$ while after it arrives, the state estimation is changed to $\Omega_{\alpha}^{\prime}$ because $\alpha$ gains additional knowledge from this token. Thus, according to Q-MDP, before $\Delta_{\text {pre }}$ is received, the expected reward of $\alpha$ is $E U\left(\Omega_{\alpha}\right)=\Omega_{\alpha} \times V$ while after the arrival of $\Delta_{\text {pre }}, E U\left(\Omega_{\alpha}^{\prime}\right)=\Omega_{\alpha}^{\prime} \times V$. Moreover, agent $\alpha$ 's local model will also be updated according to $\Delta_{\text {pre }}$. Suppose $\Delta_{\text {pre }}$ comes from acquaintance $b$ and $P_{\alpha}[\Delta, b]$ is the probability that $\alpha$ will send $\Delta$ to b before $\Delta_{\text {pre }}$ comes while $P_{\alpha}^{\prime}[\Delta, b]$ is the updated probability after the arrival of $\Delta_{\text {pre }}$. According to our assumption that policy $\pi^{* * *}$ (according to the $P_{\alpha}$ model) and $\pi^{* *}$ (according to the POMDP model) will choose the same action which is to send $\Delta$ to b. Thus, we have

$$
\frac{P_{\alpha}^{\prime}[\Delta, b]}{P_{\alpha}[\Delta, b]}=\frac{E U\left(\Omega^{\prime}{ }_{\alpha}, b\right)}{E U\left(\Omega_{\alpha}, b\right)}=\frac{\left[\Omega_{\alpha}^{\prime} \times V\right]_{b}}{\left[\Omega_{\alpha} \times V\right]_{b}}
$$

Where $\left[\Omega_{\alpha}^{\prime} \times V\right]_{b}$ is the value of the component in vector of $\left[\Omega_{\alpha}^{\prime} \times V\right]$ according to acquaintance $b$. It is the same vector as $E U\left(\Omega_{\alpha}^{\prime}, b\right)$.

According to the update function $\operatorname{Update}\left(P_{\alpha}[\Delta, b], \Delta_{\text {pre }}\right)$, we should get $P_{\alpha}^{\prime}[\Delta, b]=\operatorname{Rel}\left(\Delta, \Delta_{\text {pre }}\right) \times P_{\alpha}[\Delta, b]$. Thus, the relationship between $R e l$ and our POMDP model is:

$$
\operatorname{Rel}\left(\Delta, \Delta_{\text {pre }}\right)=\frac{\left[\Omega_{\alpha}^{\prime} \times V\right]_{b}}{\left[\Omega_{\alpha} \times V\right]_{b}}
$$

From this equation, we can conclude that a received token changes the agent's estimation of the probability distribution of the team's state, which in turn directly influences the decision of where to send related tokens. If we know a little bit of how the probability distribution changes for an agent after it has passed a token, we can use this to predict how this agent updates its distributed decision model and therefore define the relevance between tokens. A heuristic that captures this relationship will approximate the locally optimal policy and hence lead to good behavior. In this paper, we simply estimate this value based on the similarity between tokens. Intuitively, if two tokens are similar, receiving one token allows an agent to update its estimation of the team state and infer where to pass the similar tokens. For example, receiving a role token from a particular acquaintance, tells the agent that it is relatively less likely that similar role tokens will be accepted in the part of the network accessible via that acquaintance; receiving an information token with information about Pittsburgh tells the agent that some agents in that part of the network must currently be in Pittsburgh.

The similarities between tokens come from the coordination they carry and the calculation depends on the domain knowledge of applications. We assume that, from $\Delta_{i}$.coordination and $\Delta_{j}$.coordination, we can deduce the similarity between two tokens as $\operatorname{sim}\left(\Delta_{i}, \Delta_{j}\right) . \operatorname{sim}\left(\Delta_{i}, \Delta_{j}\right)>1$ if $\Delta_{i}$ and $\Delta_{j}$ are a pair of similar tokens. For example, if two tokens both reference Pittsburgh, we deem them similar because both are involved with the same location; we deem two tokens which require driving a specific machine as similar because they need the same kind of capacity; two tokens that both are preconditions of the same plan would also be considered similar.

We distinguish the relationship between relevance and similarity of two tokens as positively related or negatively related. For two similar tokens $\Delta_{i}$ and $\Delta_{j}$, if an agent previously received a token from an acquaintance and would prefer to send a similar token to that acquaintance, similar tokens are positively related to each other and $\operatorname{Rel}\left(\Delta_{i}, \Delta_{j}\right)=$ $\operatorname{sim}\left(\Delta_{i}, \Delta_{j}\right)$. Otherwise, if this agent is less likely to send the similar token to that acquaintance similar tokens are negatively related to each other, $\operatorname{so} \operatorname{Rel}\left(\Delta_{i}, \Delta_{j}\right)=\frac{1}{\operatorname{sim}\left(\Delta_{i}, \Delta_{j}\right)}$

The similarity between different types of tokens potentially influences agents' estimation in different ways. As we have shown in the previous example, receipt of role tokens discourages sending similar tokens to agents along the role tokens' paths because the previous token senders refused the role token and are incapable of accepting the role, therefore are less likely to be interested in the information, tasks or resources that similar tokens carry. Thus, a pre- 
vious role token is negatively related to its similar tokens. Similarly, receipt of an information token will indicate that agents along the information tokens' paths are more likely to work on things related to that information and are interested in other similar tokens. Hence, a previous information token is positively related to its similar tokens.

If the threshold of a resource token $\Delta_{i}$ is greater than its initial value (init) upon arrival to current agent, this means that the resource has been used by the agents previously holding $\Delta_{i}$ and that those agents are potentially engaged in tasks requiring the resource. Therefore, if the current agent gets similar tokens, it will be more likely to send them to the part of the network where the previous token has been passed. In this case, the previous resource token is positively related to similar tokens. Alternatively, if $\Delta_{i}$.threshold is lower than its initial value (init), it means that agents passing the token did not need it. In such a case, the previous resource token is negatively related to similar tokens.

Supposed $\Delta_{i}$ is a previously received token, we can summarize the calculation of $\operatorname{Rel}\left(\Delta_{i}, \Delta_{j}\right)$ according to $\operatorname{sim}\left(\Delta_{i}, \Delta_{j}\right)$. No matter what $\Delta_{j}$.Type is, this function only depends on the type of previously incoming token:

$$
\begin{aligned}
& \operatorname{Rel}\left(\Delta_{i}, \Delta_{j}\right)= \\
& \begin{cases}\operatorname{sim}\left(\Delta_{i}, \Delta_{j}\right) & \text { if } \Delta_{i} . \text { Type }=\text { inf } \\
\operatorname{sim}\left(\Delta_{i}, \Delta_{j}\right) & \text { if } \Delta_{i} . \text { Type }=\text { res, } \Delta_{i} . \text { threshold }>\text { init } \\
\frac{1}{\operatorname{sim}\left(\Delta_{i}, \Delta_{j}\right)} & \text { if } \Delta_{i} . \text { Type }=\text { role } \\
\frac{1}{\operatorname{sim}\left(\Delta_{i}, \Delta_{j}\right)} & \text { if } \Delta_{i} . \text { Type }=\text { res }, \Delta_{i} . \text { threshold }<\text { init }\end{cases}
\end{aligned}
$$

\section{EXPERIMENTAL RESULTS}

In this section, we describe an empirical evaluation of our approach. Tests were conducted using an abstract simulation called TeamSim configured to simulate a group of 400 distributed UAVs searching a hostile area. The network topology was that of a small world network where each UAV had, on average, four acquaintances. Simulating automatic detection rates, 200 pieces of information were randomly sensed by UAVs and passed around the team. Fifty plans instances, each with four independent preconditions, were given to the team. After a plan was initiated, tokens for the four roles needed to realize the plan were circulated through the acquaintance network. To accept a role an agent must be close to the region the role requires and have access to resource tokens for airspace at the role allocation. Airspace over the hostile area was divided into fifty regions. Each of these regions were duplicated in three resource tokens allowing a maximum of three UAVs to simultaneously access to that airspace. Each UAV needed to obtain the resource for the region related to its task before they could be performed. If all four roles of a plan were successfully executed, a reward of 10 units was credited to the team. A maximum reward of 500 units (10 units x 50 plan instances) was possible. Results for each experiment are based on one hundred trials.

The first experiment investigated the algorithm's performance in enhancing overall team reward. Reward obtained was recorded for each tick of the simulation which corresponded to the time taken for a token to move from agent to agent. Five configurations of the algorithm were compared. In the first configuration, agents passed tokens randomly if they did not keep them. In the next three configurations,

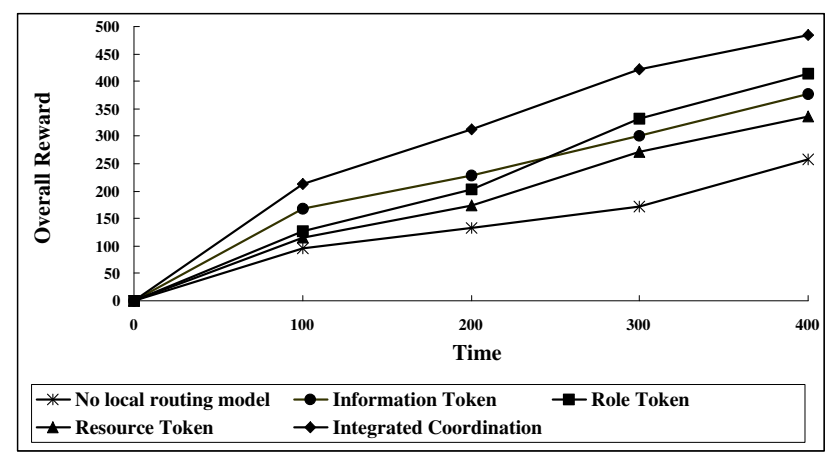

Figure 4: The team gets more reward (y-axis) over time (x-axis) when all token types are used to update local models.

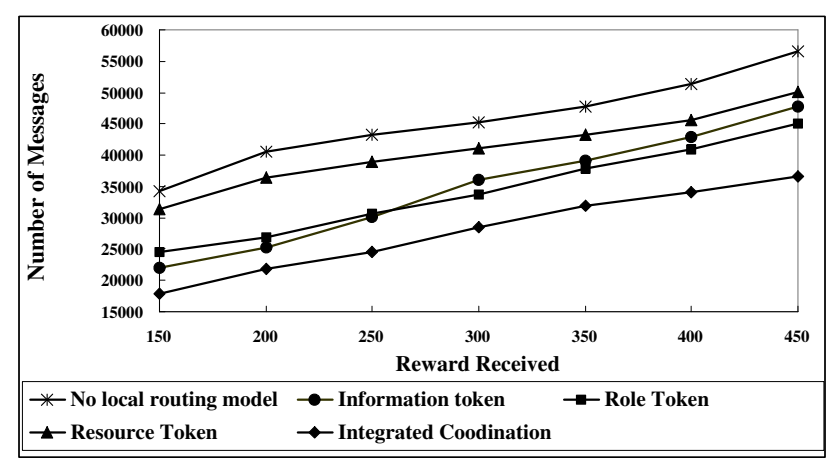

Figure 5: The team needs to send less messages (yaxis) to coordinate when all token types are used to create local models.

local reasoning model updating is only applied to only one type of token, i.e., information, resource or role, with no updating by the other two types. The fifth configuration provided integrated coordination using tokens of each type to update agents' local model for routing tokens. The results are shown in Figure 4. Use of any previous tokens for token routing improved team reward. This benefit was most pronounced when all token types were used. In fact reward was almost doubled over random token movement. Notice that early on using plan instantiation alone was more effective than using other types of tokens but later role allocation tokens were the most effective type. We hypothesize that as roles are allocated they become most useful but before then it is critical to know who is initiating plans.

The second experiment investigated the effect of our algorithm on communication. In this experiment we compared the number of messages need for a team to gain a particular level of reward. A message was credited to each transfer of a token from an agent to its acquaintance. The same five configurations (random, 3 with coordination for single token-types, and integrated) were employed. As shown in Figure 5 configurations using token coordination algorithm performed better. They used fewer communications to attain the same level of reward as the random configuration. Once again, complete integrated token routing was superior 

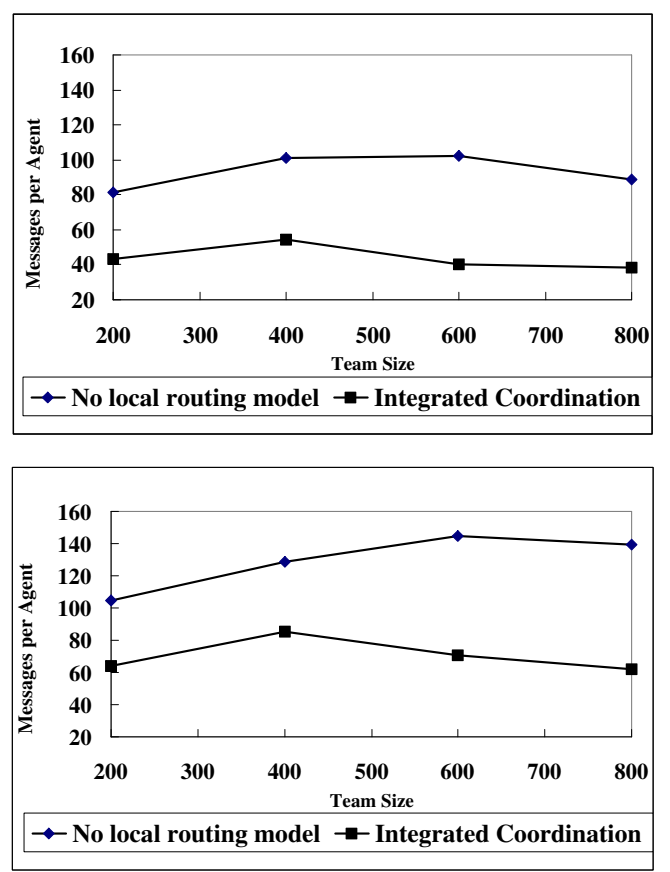

Figure 6: The average number of messages per agent (y-axis) stays relatively constant as the team size is increased (x-axis). This holds tokens when tokens are used for integrated model and when they are not.

to these partial token-based algorithm in attaining the same results with substantially fewer messages. Notice also that the total number of references is quite low, even for a large team.

The third experiment examined in more detail the scalability of our algorithm to larger teams. In this experiment teams of 200 to 800 agents were run under conditions otherwise identical to the first two experiments, however, only two configurations were used: no use of previous tokens to improve agents' local reasoning model versus use of all types of previous tokens to improve agents' local reasoning model to routing tokens. Performance was measured using average number of messages per agent. In Figure 6, the top configuration is to get a team reward of 200 units while the bottom one is to get a reward of 400 . Our results show that integrated token-based routing produced lower message overload under all conditions. For both 200 and 400 reward levels observed message overhead was lower for teams of 800 agents using the integrated algorithm than for 200 agent teams using the random one.

\section{RELATED WORK}

Multiagent coordination is an extensively studied area of multiagent systems, but most of the existing work does not scale well to very large teams. Distributed constraint-based algorithms [12, 13] have high communication requirements that get dramatically worse as the team size in increased. Combinatorial auctions[8] have an exponential number of possible combinations of bids, and frequently use centralized auctioneers that can become severe bottlenecks. Swarm- inspired approaches[3] have been used for large-scale coordination, but the behavior can be inefficient.

Decision theoretic approaches, such as MDPs and POMDPs, have been used for team coordination[7, 14, 24]. Because centralized control is frequently not possible, the team coordination problem is recast as a communication problem[14]. However, such approaches are known to be intractable in general[14], and so are of limited use in large scale applications.

Recent work focusing on scalable coordination[17] illustrates that exponential search spaces, excessive communication demands, localized views, and incomplete information of agents pose major problems for large scale systems. Initial work on token-based approaches promises a way to address these challenges. Large scale coordination in the GPGP/TAEMS framework[10] was demonstrated using a token-based algorithm[21]. The effectiveness of large-scale, token-based coordination has also been demonstrated in the Machinetta proxy architecture[16] for task allocation[15] and information sharing[23].

Research on social networks began in physics[1, 22]. [5] showed that social network structures in team formation can dramatically affect team abilities to complete cooperative tasks. In particular, using scale-free network structures for agent teams facilitates team formation by balancing the number of skill-constrained paths available in the agent organization with the effects of potential blocking. [4] compared the merits of small world networks and scale-free networks in the application of emergent coordination.

\section{CONCLUSION AND FUTURE WORK}

This paper presented a novel integrated token-based algorithm for team coordination. By utilizing relationships between tokens we were able to use the execution of one type of coordination algorithm to improve the performance of the others. Our experiments show that our approach is scalable and efficient. This approach opens the possibility to develop a range of new executing applications of heterogeneous agents not possible with existing approaches.

While the results presented here represent a step forward, they also point to significant challenges and exciting questions. We plan to address some of these issues in the near future. From a scientific perspective, the effect of the underlying acquaintance network on the coordination is clearly important but poorly understood. We will investigate the effect of the properties of the network on the team's performance, specifically looking at how the small worlds nature of the network is important. This work represented a novel attempt at integrating coordination algorithms into a unified approach and showing how by working together the overall performance can be improved. However, the individual algorithms were designed without thought to future integration. A key question is whether knowing that we will be integrating the token algorithms allows us to build algorithms that work better with other algorithms. Finally, but critically, we will use the token-based approach in more realistic domains to understand its utility in the real world. Specifically, we are currently developing large scale coordination applications for rescue response and unmanned aerial vehicle applications. 


\section{Acknowledgements}

This research has been sponsored in part by AFRL/MNK Grant F08630-03-1-0005 and AFOSR Grant F49620-01-10542 .

\section{REFERENCES}

[1] A. Barabasi and E. Bonabeau. Scale free networks. Scientific American, pages 60-69, May 2003.

[2] A. Cassandra, M. Littman and N. Zhang Incremental pruning: a simple, fast, exact method for partially observable Markov decision processes. Proceedings of the Thirteenth Annual Conference on Uncertainty in Artificial Intelligence, 1997.

[3] V. Cicirello and S. Smith. Wasp nests for self-configurable factories. In Proceedings of the Fifth International Conference on Autonomous Agents, 2001.

[4] J. Delgado, J. Pujol and R. Sanguesa. Emergence of coordination in scale-free networks. In $W e b$ Intelligence and Agent Systems 131-138, 2003.

[5] M. Gaston and M. desJardins. Social network structures and their impact on multi-agent system dynamics. In Proceedings of the 18th International Florida Artificial Intelligence Research Society Conference, 2005.

[6] D. Goldberg, V. Cicirello, M. Dias, R. Simmons, S. Smith, and A. Stentz. Market-based multi-robot planning in a distributed layered architecture. In International Workshop on Multi-Robot Systems. Kluwer Academic Publishers, 2003.

[7] C. Goldman and S. Zilberstein. Optimizing information exchange in cooperative multi-agent systems. In Proceedings of the Second International Joint Conference on Autonomous Agents and Multiagent Systems, 2003.

[8] L. Hunsberger and B. Grosz. A combinatorial auction for collaborative planning. In Proceedings of the Fourth International Conference on Multi-Agent Systems, pages $151-158,2000$.

[9] H. Kitano, S. Tadokoro, I. Noda, H. Matsubara, S. Takahashi, A. Shinjoh and S. Shimada. Robocup rescue: Search and rescue in large-scale disasters as a domain for autonomous agents research. In IEEE International Conference on Systems, Man and Cybernetics, volume VI, pages 739-743, 1999.

[10] V. Lesser, K. Decker, T. Wagner, N. Carver, A. Garvey, B. Horling, D. Neiman, R. Podorozhny, M. NagendraPrasad, A. Raja, R. Vincent, P. Xuan and X. Zhang. Evolution of the GPGP/TEAMS domain-independent coordination framework. Autonomous Agents and Multi-Agent Systems, 9, 2004.

[11] M. Littman, A. Cassandra, and L. Kaelbling. Learning policies for partially observable environments: scaling up. In International Conference on Machine Learning, 1995.

[12] R. Mailler and V. Lesser. Solving distributed constraint optimization problems using cooperative mediation. In Proceedings of Third International Joint Conference on Autonomous Agents and Multiagent Systems, 2004.

[13] P. Modi, W. Shen, M. Tambe and M. Yokoo. An asynchronous complete method for distributed constraint optimization. In Proceedings of Second International Joint Conference on Autonomous Agents and Multiagent Systems, 2003.

[14] D. Pynadath and M. Tambe. The communicative multiagent team decision problem: analyzing teamwork theories and models. In Journal of AI research, 2002.

[15] P. Scerri, A. Farinelli, S. Okamoto and M. Tambe. Allocating tasks in extreme teams. In Proceedings of Fourth International Joint Conference on Autonomous Agents and Multiagent Systems, Forthcoming, 2005.

[16] P. Scerri, D. Pynadath, L. Johnson, P. Rosenbloom, N. Schurr, M. Shi and M. Tambe. A prototype infrastructure for distributed robot-agent-person teams. In Proceedings of Second International Joint Conference on Autonomous Agents and Multiagent Systems, 2003.

[17] P. Scerri, R. Vincent and R. Mailler. Comparing three approaches to large scale coordination. In $A A M A S^{\prime} 04$ Workshop on Challenges in the Coordination of Large Scale MultiAgent Systems, 2004.

[18] P. Scerri, Y. Xu, E. Liao, J. Lai and K. Sycara. Scaling teamwork to very large teams. In Proceedings of Third International Joint Conference on Autonomous Agents and Multiagent Systems, 2004.

[19] M. Tambe. Towards flexible teamwork. Journal of AI research, 7: 83-124, 1997.

[20] A. Vick, R. Moore, B. Pirnie and J. Stillion. Aerospace operations against elusive ground targets. RAND Documents, 2001.

[21] T. Wagner, V. Guralnik and J. Phelps. A key-based coordination algorithm for dynamic readiness and repair service coordination. In Proceedings of Second International Joint Conference on Autonomous Agents and Multiagent Systems, 2003.

[22] D. Watts and S. Strogatz. Collective dynamics of small world networks. Nature, 393: 440-442, 1998.

[23] Y. Xu, M. Lewis, K. Sycara and P. Scerri. Information sharing in large scale teams. In $A A M A S^{\prime}{ }_{4}$ Workshop on Challenges in Coordination of Large Scale MultiAgent Systems, 2004.

[24] P. Xuan, V. Lesser and S. Zilberstein. Communication decisions in multi-agent cooperation: Model and experiments. In Proceedings of the Fifth International Conference on Autonomous Agents, 2001. 